\title{
Pour une approche interculturelle dans l'étude de la relation entre les bénévoles et les communautés hôtes dans le bénévolat international
}

\author{
Mathilde Gouin-Bonenfant ${ }^{\mathrm{a}}$
}

RÉSUMÉ. Les écrits sur la relation entre les bénévoles et les communautés hôtes dans le bénévolat international sont partagés entre deux postures opposées. La première, enthousiaste, soutient que le bénévolat international favorise un meilleur rapprochement interculturel que le tourisme. La seconde, critique, y met de l'avant la reproduction des rapports de pouvoir postcoloniaux. Pour surpasser cette opposition, cet article propose un cadre théorique interculturel, inspiré de la philosophie herméneutique de Gadamer, des approches systémiques des relations humaines, de l'ethnographie de la communication et de la sociolinguistique interactionnelle. Pour illustrer l'apport de celui-ci, une étude de cas sur la relation entre les stagiaires et leurs familles d'accueil dans les stages Québec Sans Frontières au Sénégal sera présentée. Celle-ci mettra en lumière trois dynamiques concomitantes dans cette relation : la (co)production de savoirs culturels, la négociation de nouveaux systèmes de communication hybrides et temporaires et le développement de savoir-faire interculturels.

\begin{abstract}
$\boldsymbol{A B S T R A C T}$. The literature on the relationship between volunteers and host communities in international volunteering is divided into two opposing positions. The first is enthusiastic and argues that international volunteering promotes better intercultural relations than tourism. The second is more critical and promotes the reproduction of postcolonial power relations. To surpass this opposition, this article proposes an intercultural theoretical framework. inspired by Gadamer's hermeneutic philosophy, systemic approaches to buman relations, communication ethnography and interactive sociolinguistics. To illustrate the contribution of this framework, we will present a case study on the relationship between volunteers and their host families in "Quebec Sans Frontières" internships in Senegal. The case study will highlight three concomitant dynamics in this relationship: the (co-)production of cultural knowledge, the negotiation of new bybrid and temporary communication systems and the development of intercultural know-how.
\end{abstract}

\section{Introduction}

Depuis quelques années, les critiques du volontourisme se multiplient dans les médias, à travers le monde ${ }^{1}$. En 2016, plusieurs voix se sont élevées au Québec pour dénoncer le volontourisme en l'accusant d'être une forme de tourisme centrée sur le profit et qui ferait plus de mal que de bien aux communautés locales (Gagnon, 2016; Hachey, 2016; Lawrence, 2016). C'est un article dans La Presse qui a débuté ce débat. Celui-ci traite des expériences de volontourisme avec la compagnie Projects Abroad. Les séjours qui y sont organisés sont décrits comme étant au mieux inutiles - l'auteure donne l'exemple de bénévoles qui repeignent la même murale à chaque visite - au pire très nuisibles. Au Cambodge, là où la journaliste a mené ses enquêtes, les orphelinats prolifèrent pour répondre à la demande en ce sens, alors que les séjours de courtes durées des bénévoles y auraient des répercussions négatives sur les enfants (Hachey, 2016).

$\mathrm{Au}$ cœur des critiques du volontourisme se retrouve souvent la relation entre les communautés hôtes et les bénévoles. C'est aussi l'objet d'une importante partie de la littérature académique sur ce thème. Les bénévoles causent-ils plus de mal que de bien aux communautés où ils et elles séjournent?

a M. Sc. Département d'anthropologie, Université de Montréal 
Le volontourisme reproduit-il des rapports de pouvoir asymétriques? Quelles sont les réelles motivations des bénévoles? Le volontourisme permet-il un meilleur rapprochement interculturel que le tourisme "ordinaire »? D'un côté, certain-e-s auteur-e-s, se positionnant dans une posture enthousiaste, vont affirmer que le volontourisme est caractérisé par une meilleure compréhension interculturelle, par l'empowerment des bénévoles et des communautés hôtes, par le dialogue, la tolérance, le rapprochement, l'action sociale, et un sens de citoyenneté du monde (Coghlan et Gooch, 2011; Crabtree, 1998; Higgins-Desbiolles, 2003; Mcgehee, 2002; Mcgehee et Santos, 2004; Wearing, 2001). De l'autre côté, d'autres études montrent que ces programmes peuvent aussi mener au renforcement des stéréotypes, à de mauvaises compréhensions de l'Autre et à la reproduction de rapports de pouvoir néocoloniaux (Daldeniz et Hampton, 2010; Gius, 2012; Guttentag, 2009; Raymond et Hall, 2008; Simpson, 2004). Cet article propose de sortir de cette dichotomie - en déplaçant l'objet d'étude du résultat de la rencontre à son processus. Pour effectuer ce déplacement, il faut alors développer un cadre théorique et méthodologique interculturel.

Dans la littérature sur le bénévolat international, la différence entre le bénévolat organisé par des ONG et celui organisé par des entreprises privées est rarement explicitée. De plus, le terme «volontourisme ", souvent préconisé, ne permet pas cette contextualisation. Cet article offrira donc premièrement un éclaircissement autour des termes de volontourisme et de bénévolat international, autour de cette distinction. Ensuite, l'opposition entre les postures enthousiastes et critiques - les deux postures dominantes - sera présentée. Pour dépasser cette dichotomie, cet article soulignera alors une série de problèmes qui participe ou découle de cette opposition, pour ensuite mettre de l'avant un cadre théorique interculturel. Finalement, pour illustrer l'apport d'une telle posture, une étude sur la relation entre les stagiaires et leurs familles d'accueil dans les stages Québec Sans Frontières (QSF) au Sénégal sera présentée ${ }^{2}$.

\section{Volontourisme et bénévolat international}

Il existe dans la littérature académique, comme dans l'usage courant, une confusion autour du concept de volontourisme. Évoquant un lien de parenté avec le tourisme et le bénévolat (volontariat), ce terme est aussi de plus en plus connoté négativement. Il convient ainsi de démêler les concepts de tourisme, de bénévolat international et de volontourisme.

En Europe, c'est au 19e siècle, grâce aux innovations technologiques dans le transport et la médecine, que se développe le tourisme (Van den Berghe, 1980). L'industrie touristique, quant à elle, nait après la Seconde Guerre mondiale, lorsque les classes moyennes d'Europe et d'Amérique du Nord acquièrent des congés payés et peuvent alors se déplacer en profitant des nouveaux moyens de transport (Colleyn et Devillez, 2009). C'est au même moment, après la Deuxième Guerre mondiale, qu'émerge le développement international. Une de ces facettes, c'est le bénévolat international pour le développement. Après la Deuxième Guerre mondiale, des organismes tels Voluntary Service Overseas au Royaume-Uni et Peace Corps aux États-Unis voient le jour (Butcher et Smith, 2010). Ce phénomène, qui regroupe mobilité, engagement bénévole de jeunes adultes et développement international n'est cependant pas étiqueté, à l'époque, comme étant une forme tourisme (Daldeniz et Hampton, 2010).

Il faut attendre encore quelques décennies avant que l'association entre bénévolat international et tourisme soit établie. Durant les années 1980, le tourisme est en forte croissance. En réaction, le concept de «tourisme alternatif» nait, d'une volonté de transformer le rapport entre les communautés hôtes et les touristes (McIntosh et Zahra, 2007; Stronza, 2001). L'exemple le plus commun et étudié est celui de l'écotourisme, soit une forme de tourisme qui s'intéresse aux écosystèmes naturels et culturels (Stronza, 2001; Lyons et Wearing, 2008). Durant les années 1990, les Organisations non gouvernementales (ONG) sont en forte croissance. Témoins de la popularité des formes de tourisme alternatif, les ONG développent des programmes de bénévolat international répondant à leurs valeurs et leurs missions (Lyons et Wearing, 2008). Les années 2000 voient ensuite l'apparition de grandes organisations qui commercialisent les expériences de bénévolat international. Dans le contexte néolibéral ambiant, le volontourisme devient alors une industrie, à l'image de l'industrie touristique (Lyons, Hanley, Wearing et Neil 2012). C'est donc à l'intersection de l'industrie touristique 
et du bénévolat international que nait, à notre époque néolibérale, l'industrie volontouristique.

Selon McIntosh et Zahra (2007) le tourisme bénévole (volontourisme) inclut l'expérience de " gap year » au Royaume-Uni et en Australie, de petites " missions » internationales, la participation à des recherches environnementales, ou les échanges culturels (McIntosh et Zahra, 2007, p. 543). Ainsi, la durée, le coût, l'encadrement, la nature des projets, les formations prédépart et les conditions de vie sur le terrain varient énormément dans ces différentes expériences. Lyons et Wearing (2008), sans employer des termes différents pour les deux phénomènes, font tout de même une distinction entre le tourisme bénévole organisé par les ONG et celui organisé par des entreprises pour le profit. Selon eux, les ONG, étant donné leur logique non marchande cultivent de meilleures pratiques que les entreprises dont l'idéologie capitaliste place le profit avant les humains (Lyons et Wearing, 2008).

En français, le terme « volontourisme » semble se populariser avec l'émergence des entreprises privées qui commercialisent le bénévolat international dans les années 2000. En anglais, toutefois, cette distinction existe moins clairement, puisque c'est à partir du terme « volunteer tourism» (tourisme bénévole) qu'a été créé celui de « voluntourism » (volontourisme). Les deux termes sont maintenant souvent utilisés comme des synonymes dans la littérature académique (Pastran, 2014; McAllum et Zahra, 2013). Ils sont par ailleurs employés pour désigner un éventail de phénomènes qui regroupent le tourisme alternatif, le bénévolat international organisé par les ONG, ainsi que par des entreprises privées.

Malgré que les écrits universitaires sur le bénévolat international ne font donc pas (ou peu) la différence entre le bénévolat international organisé par des ONG et celui organisé par des entreprises commerciales, il existe tout de même une différence majeure entre les deux, soit l'opposition entre une logique marchande et une logique de solidarité. Le choix du terme de "bénévolat international» permet ainsi d'échapper à la confusion que peut amener celui de "volontourisme». Le bénévolat international est alors caractérisé par 1) un déplacement, 2) temporaire, 3) dont le but est le travail bénévole. Il se sous-divise ensuite entre le bénévolat international organisé par des ONG et le bénévolat international industrialisé.

\section{Une littérature divisée}

Les écrits sur le bénévolat international se sont considérablement accrus dans les années 2000, au même moment où le bénévolat international commercialisé prenait son essor. Celui-ci reste toutefois, selon plusieurs auteur-e-s, sous-théorisé (Butcher et Smith, 2010; Daldeniz et Hampton, 2010). Puisque le bénévolat international est souvent conçu comme une forme de tourisme alternatif (Wearing, 2001; Wearing et Mcgehee, 2013), les écrits sur ce phénomène ont repris les thématiques, les concepts et les postures de la littérature sur le tourisme. En effet, c'est ce que Wearing et McGehee (2013) démontrent en reprenant les étapes du développement de la littérature sur le tourisme de masse, mis en lumière par Jafari (2001). Il s'agit de la défense, la précaution, l'adaptation et la posture scientifique (Wearing et McGehee, 2013). Cette progression n'est toutefois pas nécessairement linéaire et ce qui aura duré plusieurs décennies pour l'étude du tourisme de masse s'est plutôt déroulé en quelques années pour le bénévolat international (Wearing et McGehee, 2013).

Initialement, donc, la majorité de la recherche sur le bénévolat international défendait et faisait la promotion de ce phénomène, décrit comme une forme de tourisme alternatif, motivé par l'altruisme et menant à de meilleures compréhensions mutuelles. Cette posture de défense a alors peu à peu cédé la place à une posture critique dans laquelle les impacts négatifs du bénévolat international étaient aussi présentés. Une posture d'adaptation apparait depuis les années 2010, proposant de bonnes pratiques pour maximiser les impacts positifs du bénévolat international. Finalement, la posture scientifique, quatrième étape, serait déjà amorcée. Il s'agirait d'une étude plus systématique, logique, structurée, transdisciplinaire, internationale du bénévolat international, à partir des fondements théoriques explicites (Wearing et McGehee, 2013). Cette analyse semble juste, malgré que les deux premières postures soient toujours prédominantes. Suivant McAllum et Zahra (2017), nous les nommerons postures « enthousiastes » et « critiques ».

La posture enthousiaste est orientée vers plusieurs idéaux ou promesses. Ainsi, le bénévolat international est tour à tour décrit comme un tourisme plus moral ou plus juste (Butcher, 2003); une expérience qui engendre des impacts positifs sur les 
bénévoles et qui peut mener à des transformations de leurs valeurs (Broad, 2003; Coghlan et Gooch, 2011; Crabtree, 1998; Mcgehee, 2002; Mcgehee et Santos, 2004; McIntosh et Zahra, 2007; Wearing, 2001); un phénomène qui a des impacts positifs à la fois sur les bénévoles et les communautés hôtes (McIntosh et Zahra, 2007); qui provoque un accroissement de l'engagement social des bénévoles (Mcgehee, 2002; Mcgehee et Santos, 2004); qui permet le développement d'un sentiment de citoyenneté mondiale (Crabtree, 1998); qui permet un rapprochement interculturel ou une meilleure compréhension mutuelle entre les bénévoles et communautés hôtes (Broad, 2003; Crabtree, 1998; McIntosh et Zahra, 2007; Wearing, 2001); et finalement, qui permet même la réconciliation (Crabtree, 1998; Higgins-Desbiolles, 2003).

Comme dans les écrits sur le tourisme, cette posture est toutefois remise en question, en premier lieu par des auteur-e-s qui se méfient du présupposé que le bénévolat international n'a que des effets positifs, puis par des critiques postcoloniales qui vont critiquer les structures permettant cette mobilité et les rapports de domination qui y sont reproduits.

Plusieurs auteur-e-s ont donc voulu démontrer que le bénévolat international n'a pas que des effets positifs. Guttentag (2009), dans un article intitulé "The possible negative impacts of volunteer tourism », met ainsi en lumière une série d'impacts négatifs potentiels. Il identifie par exemple la négligence des volontés et désirs des populations locales, l'insatisfaction vis-à-vis de la réalisation du travail, causée par le manque de compétences des bénévoles, une diminution des opportunités d'emploi pour les populations locales, dû au travail des bénévoles, ainsi que le renforcement des stéréotypes des bénévoles (Guttentag, 2009). L'auteur ne remet pas en question, toutefois, que le bénévolat international puisse aussi avoir des effets positifs. C'est le présupposé qu'il n'y a que des effets positifs qui est critiqué. Plusieurs autres auteur-e-s s'inscrivent aussi dans cette remise en question des bienfaits du bénévolat international (Daldeniz et Hampton, 2010; Lyons et al, 2012; Sin, 2009).

À l'instar du tourisme, le bénévolat international est aussi l'objet de fortes critiques postcoloniales. Ce qui est intéressant dans le cas du bénévolat international, c'est qu'il est souvent conceptualisé à la croisée entre le tourisme et le développement international. Or, selon plusieurs auteur-e-s postcoloniaux, le développement international est un projet impérial et néocolonial de l'Occident (Pastran, 2014). Il est intéressant de noter que le tourisme de masse et le développement international ont émergé en parallèle l'un de l'autre, à la suite de la Deuxième Guerre mondiale, et qu'ils empruntent les mêmes trajectoires de mobilité et les mêmes structures de privilèges. Le bénévolat international est ainsi doublement critiqué. Selon les critiques, ce serait une forme de néocolonialisme qui ne ferait que renforcer les relations inégales de pouvoir entre les bénévoles et les communautés hôtes (Pastran 2014). En plus d'avoir une posture de méfiance, plusieurs études vont donc adopter une posture postcoloniale et démontrer que les dynamiques de pouvoir et de privilèges sont renforcées et reproduites dans la rencontre entre les communautés hôtes et les bénévoles.

Simpson (2004) fait ressortir la «mythologie du développement » présentée dans le matériel de promotion d'expériences de bénévolat international, dans laquelle le "tiers-monde » est conçu comme ayant besoin d'aide et les jeunes européens comme ayant l'habileté et le droit de répondre à ces besoins. Charia Gius (2012) à la suite d'une étude de terrain, conclut que l'expérience de bénévolat international radicalise la conception de l'altérité, en mettant l'accent sur les différences plutôt que sur les points communs (Gius 2012). Smith et Laurie (2011) mettent de l'avant la violence systémique qui caractérise la domination d'une géographie de la mobilité et d'un imaginaire spatial dans le bénévolat international (Smith et Laurie, 2011). Mostafanezhad (2014) critique plutôt le « regard populaire humanitaire » dont est investi le bénévolat international et qui participe à mettre de l'avant un discours géopolitique sur les rapports nord-sud dans lequel les relations politiques et économiques, ainsi que les inégalités sociales, deviennent naturelles et apolitiques. Ces discours sur les relations Nord-Sud ne permettent pas d'historiciser la pauvreté et les rapports de domination et mettent plutôt de l'avant la rencontre interculturelle et son esthétique (Mostafanezhad, 2014).

Les écrits sur le bénévolat international sont donc dominés par une opposition entre les postures enthousiastes et critiques. Toujours selon les étapes de Jafari (2001) et l'analyse qu'en ont faite Wearing 
et McGehee (2013), il y aurait deux autres postures aussi présentes, soit la posture d'adaptation et celle dite scientifique. La première proposerait de «bonnes pratiques» pour maximiser les impacts positifs du bénévolat international. Plusieurs recherches s'inscrivent effectivement dans cette approche (Coghlan et Gooch, 2011; Sin, 2010; Theerapappisit, 2009). La dernière posture est celle dite scientifique. Wearing et McGehee (2013) écrivent qu'il s'agit d'une étude plus systématique, logique, structurée, transdisciplinaire, internationale du volontourisme, à partir de fondements théoriques explicites, comme les théories féministes ou les théories du développement (Wearing et McGehee, 2013). Il ne semble pas, toutefois, que ces postures soient devenues dominantes et permettent de sortir de l'opposition entre les postures enthousiastes et critiques.

\section{Trois problèmes dans la littérature}

L'opposition entre ces deux postures nous amène à une impasse qui rend difficile le renouvellement des écrits sur le bénévolat international. Pour sortir de celle-ci, cet article propose de mettre en lumière des problématiques qui perpétuent ou qui découlent de cette opposition. Cela nous permettra ensuite de proposer une troisième approche.

Le premier problème identifié est celui du manque de contextualisation. Il semble que les études sur les relations entre communautés hôtes et bénévoles se posent continuellement la question de savoir si de telles expériences sont bénéfiques ou nuisibles, ou si le volontourisme permet (ou non) un meilleur rapprochement interculturel que le tourisme de masse. Les auteur-e-s répondent, en se basant sur des études de cas radicalement différentes les unes des autres. Comme nous en avons fait la démonstration, le terme "volontourisme » est utilisé pour désigner une très grande variété d'expériences qui peuvent être très différentes les unes des autres. Or, dans l'étude de la rencontre, la question du contexte est importante. Dans une analyse systémique, nous pouvons affirmer que les interactions et relations entre les individus constituent un système ouvert. Celui-ci est influencé et influence son contexte. Les individus, leurs relations et le contexte constituent alors un deuxième système, à une autre échelle d'analyse (Marc et Picard, 2000). C'est pourquoi la différence entre le bénévolat international commercialisé et celui organisé par des ONG est importante à souligner. Si la différence des résultats est aussi grande, c'est entre autres parce que le regroupement de toutes ces formes de mobilité sous l'appellation de «volontourisme» ne permet pas de répondre à la question.

Le deuxième problème identifié est celui de la passivité attribuée aux communautés hôtes. En effet, l'une des critiques récurrentes des analyses de la relation entre touristes et communautés hôtes est que, très souvent, un seul côté de la rencontre est étudié (Stronza, 2001). Rares sont les études qui interrogent le point de vue des communautés hôtes. Si cette tendance est lourde dans les postures enthousiastes, elle l'est aussi dans les postures critiques. En effet, les auteur-e-s qui s'inscrivent dans ces postures vont souvent s'attaquer à la reproduction des rapports de pouvoir dans les actions et discours des bénévoles, sans toutefois prendre en compte les points de vue des communautés hôtes. C'est comme s'il était inféré que les rapports de pouvoir macro détermineraient les interactions à l'échelle micro et empêcheraient les communautés locales d'avoir quelle qu'agentivité que ce soit dans leur relation avec les bénévoles. Effectivement, il est souvent affirmé que les relations entre communautés hôtes et bénévoles sont intrinsèquement inégales (Pastran, 2014; Lyons et al., 2012), figeant les premières dans une posture de victime.

Cette victimisation est problématique en soi, mais elle n'est pas non plus intéressante du point de vue des dynamiques qui ne peuvent (ou pas) être abordées. Par exemple, elle ne permet pas de reconnaître, ni d'étudier, l'apport des communautés hôtes dans la rencontre, ou les stratégies employées qu'elles choisissent pour prendre avantage du volontourisme. McAllum et Zahra (2013) partent de cette critique et proposent plutôt d'étudier l'agentivité des communautés dans leurs représentations des bénévoles. Leurs résultats montrent que celles-ci attribuent différentes identités aux bénévoles (identité exotique, identité d'amitié, de modèle) qu'elles mobilisent alors stratégiquement (McAllum et Zahra, 2013, p. 125). Ainsi, en reconnaissant les membres des communautés hôtes comme acteurs et actrices de la rencontre, les dynamiques qui peuvent être étudiées se multiplient.

L'étude de McAllum et Zahra (2013) se démarque aussi de la majorité des autres études de cas, 
puisque son objet d'étude n'est pas le résultat de l'expérience de bénévolat international. C'est plutôt le processus de production des représentations des bénévoles par les membres de la communauté hôte qui est l'objet d'étude. C'est que les auteures sont dans une démarche interactionniste. Elles s'intéressent à comment (par quelles stratégies) les représentations des bénévoles sont produites, mobilisées et comment celles-ci évoluent dans le temps. Le troisième problème identifié est précisément cet accent mis sur le résultat de la rencontre. Les mêmes auteures ont aussi publié, en 2017, un article sur le processus d'altérisation (othering), toujours en se concentrant sur le point de vue des communautés hôtes et en analysant le processus plus que le résultat. Selon elles, les postures enthousiastes ne remettent pas en question les bienfaits du bénévolat international, alors que les postures critiques abordent la rencontre uniquement comme un lieu de lutte de domination et de contrôle (McAllum et Zahra, 2017). Lorsque ces derniers écrits abordent l'altérisation, c'est uniquement pour démontrer comment les bénévoles instrumentalisent «l'Autre » dans leur processus de reconstruction du Soi. Cet angle d'analyse ne reconnaitrait pas la nature interactionnelle des processus d'altérisation (McAllum et Zahra, 2017). Les résultats de leur recherche soulignent trois moments dans ce processus. En premier lieu, les membres de la communauté, ainsi que les bénévoles, vont mutuellement se situer comme étant fondamentalement différents. Cette première étape s'accompagne d'une distance entre les deux groupes et d'une asymétrie. Dans un deuxième temps, les membres des communautés hôtes deviennent conscient-e-s des difficultés d'adaptation des bénévoles, en particulier dans leur incapacité à décoder les règles culturelles. Les faux pas culturels des bénévoles permettent alors de réduire la distance sociale et de rétablir un sens d'égalité. Dans un troisième temps, les membres des communautés ont transformé leur notion de Soi, en relation avec les bénévoles. Ainsi, ils ont pris les rôles de guides ou de mères, renversant alors la relation d'aide. Cette étude permet à la fois de reconnaitre l'agentivité des membres des communautés hôtes, et d'étudier le processus d'altérisation qui leur permet d'acquérir un statut supérieur et d'influencer les bénévoles à transformer leurs comportements (McAllum et Zahra, 2017).

\section{Vers une approche interculturelle : le cas des stages Québec Sans Frontières}

La reproduction de l'opposition entre les postures enthousiastes et critiques proviendrait ainsi de plusieurs écueils. Nous avons identifié le manque de contextualisation, la passivité imposée aux communautés hôtes et l'étude du résultat de la rencontre et non de son processus. Dans les trois cas, il s'agit d'une non-reconnaissance de la nature interactionnelle de la relation entre les communautés hôtes et les bénévoles. Je propose donc, pour sortir de cette opposition, une approche théorique interculturelle. Afin d'illustrer l'apport d'une telle approche, nous nous intéresserons à un cas précis, soit la relation entre les bénévoles et leurs familles d'accueil dans les stages Québec Sans Frontières (QSF) au Sénégal. Celle-ci a été l'objet de mon mémoire «Du choc à la confusion : La rencontre interculturelle dans les stages Québec Sans Frontières au Sénégal » (Gouin-Bonenfant, 2018).

Suivant la nécessité de contextualisation identifiée précédemment, il sera premièrement question du programme QSF. Celui-ci, créé en 1995, permet à des dizaines de jeunes Québécois-e-s, chaque année, de séjourner dans des communautés « du Sud ", pour y réaliser des stages d'initiation à la coopération internationale. À l'intérieur de ces expériences, les stagiaires sont logés dans des familles d'accueil et vivent le plus souvent dans des communautés rurales. Ils et elles travaillent bénévolement avec des organismes locaux. Le programme QSF se défend d'être du volontourisme et propose plutôt l'appellation « solidarité internationale » ou «initiation à la coopération internationale » (Venne, 2016). Le programme est cogéré par le ministère des Relations internationales et de la Francophonie (MRIF) et l'Association québécoise des organismes de coopération internationale (AQOCI) et les stages sont organisés par les différents organismes de coopération internationale. Les stages QSF s'inscrivent ainsi dans le phénomène de bénévolat international organisé par des ONG avec une logique qui se veut solidaire. L'accent est aussi mis, dans le programme, sur le rapprochement interculturel. En effet, une des valeurs du programme QSF est de "[favoriser] la création de liens privilégiés entre les jeunes du Québec et les populations du Sud » (QSF, 2018). 
En partant des problématiques identifiées dans la littérature, cette étude de cas s'est intéressée à la rencontre entre les bénévoles et leurs familles d'accueil comme un processus dynamique et interactionnel. Alors que le bénévolat international est souvent étudié avec un appareillage théorique et des analyses empruntées aux études touristiques, cette recherche s'inspire plutôt de la littérature sur la mobilité étudiante qui met l'accent surtout sur l'adaptation, les compétences et les apprentissages développés à travers l'expérience de mobilité (Bartel-Radic, 2014; Bathurst et La Brack, 2010; Dettweiler et al., 2015; Murphy-Lejeune, 2000; Ward et Kennedy, 1999). Cette grille de lecture a permis de reconnaitre l'importance de la production des savoirs culturels et interculturels dans le processus d'adaptation des stagiaires. C'est donc sur ces derniers que s'est concentrée la recherche.

\subsection{Un cadre théorique interculturel}

Pour parvenir à étudier comment se (co-)produisent les savoirs culturels et interculturels dans la rencontre, il m'a fallu développer une approche théorique interactionniste et interculturelle. Celle-ci a quatre sources d'inspiration: la philosophie herméneutique de Gadamer, les approches systémiques des relations humaines, l'ethnographie de la communication et la sociolinguistique interactionnelle ${ }^{3}$.

La philosophie herméneutique de Gadamer est une philosophie de l'interprétation (Grondin, 2017). Elle peut se concevoir comme une grille de lecture qui permet de penser la compréhension avec l'Autre, à travers les notions de préjugé, de tradition, de cercle herméneutique et de fusion des horizons. Dans celleci, les individus s'inscrivent dans des traditions. Les traditions seraient le « travail de l'histoire », «l'historicité de chaque individu qui influe sur sa compréhension. » (Debono, 2012, p. 155). Il s'agit du point à partir duquel les individus voient et comprennent le monde (Agar, 1982).

Les " préjugés » sont pour leur part des informations qui ne sont pas encore validées (Agar, 1982). Gadamer en 1960 écrit : «En soi, préjugé veut dire jugement porté avant l'examen définitif de tous les éléments déterminants quant au fond " (Gadamer, 1996, p. 291). Le préjugé n’est alors ni négatif en soi, ni une entrave à la compréhension. Les préjugés sont en fait la condition même de la compréhension, puisqu'ils constituent notre "précompréhension» (Gadamer, 1996, p. 298).
Alors que pour les lumières, la pensée est linéaire, Gadamer (suivant Heidegger) affirme que la compréhension est circulaire (Grondin, 2005). Le cercle herméneutique est alors un aller-retour entre le préjugé et la compréhension, mais aussi entre la partie et le tout (Grondin, 2002). C'est donc le mouvement du préjugé à la compréhension, par l'interprétation et de la compréhension nouvelle au préjugé modifié, par la révision. Ainsi, comprendre, c'est comprendre à partir de sa tradition, avec ses préjugés (Descheneaux, 2014). Or, en comprenant, on transforme ces derniers et on transforme nos attentes de compréhension. Dès lors, "dès que l'on comprend, on comprend autrement» (Gadamer, 1996, p. 318).

L'horizon est ce qui est perçu à partir d'un point de vue (Grondin, 2005). Dans les mots de Gadamer (1960), c'est « [...] le champ de vision qui comprend et inclut tout ce que l'on peut voir d'un point précis » (Gadamer, 1996, p. 324). L'horizon diffère alors d'une personne à une autre en fonction entre autres, des traditions dans lesquelles elles s'inscrivent. Pour Gadamer, la fusion des horizons « ne signifie pas non plus une unité stable et identifiable, mais quelque chose qui arrive à la faveur d'un dialogue qui se poursuit toujours. » (Grondin, 2005, p. 1). La fusion des horizons peut donc se comprendre comme un processus de réduction des écarts entre les compréhensions des individus dans lequel, ponctuellement, des significations communes sont produites.

L'approche systémique de l'école Palo Alto propose de penser les relations humaines comme des systèmes. Dans les systèmes humains, « [...] les objets qui constituent les éléments du système [sont] les individus en interactions. Les attributs qui sont les propriétés de ces objets [sont] leurs actions et leurs réactions, leurs attitudes, leurs rôles ou, d'une manière plus générale, leurs comportements.» (Marc et Picard, 2000, p. 23). Les systèmes humains sont aussi des systèmes ouverts, c'est-à-dire que les systèmes sont en communication avec leurs contextes et que chaque système s'insère dans un autre système plus large. L'échelle d'analyse n'est pas l'individu, mais la totalité, et l'unité d'analyse est l'interaction comme processus, inscrit dans un contexte. Cette approche permet donc de penser les interactions entre les individus et entre l'individu et son environnement (Marc et Picard, 2000). Elle corrobore aussi la nécessité de contextualiser les expériences de bénévolat international et de bien situer l'échelle d'analyse préconisée. 
L'ethnographie de la communication et la sociolinguistique interactionnelle sont deux approches s'inscrivant dans une analyse systémique de la communication. L'ethnographie de la communication s'intéresse aux comportements communicatifs (Hymes, 1964). Dans cette approche, la parole prime donc sur la langue, comme la fonction de la parole prime sur sa structure, et le contexte sur le message (Hymes, 1964). Les pratiques communicatives, dans une communauté donnée, se constituent alors comme des systèmes de communications. Ceux-ci ne sont pas fermés et perméables, mais sont en rapport constant avec les individus et les contextes. Les individus peuvent alors être plus ou moins compétents dans ceux-ci. Il ne s'agit pas ici de compétences linguistiques, mais plutôt de compétences communicatives (Farah, 1997).

La sociolinguistique interactionnelle s'intéresse entre autres à la création de sens dans l'interaction et à l'influence des pratiques linguistiques sur le résultat des interactions interculturelles (Tannen, 2005). Dans l'interaction, les interprétations sont construites sur des savoirs contextuels spécifiques, sous forme de présuppositions (Gumperz, 2005). Les individus sont socialisés à associer des ressources communicationnelles particulières à des cadres d'interprétation spécifiques. La façon d'inférer, dans l'interaction, est donc différente d'un groupe à l'autre (Heller, 2013). La sociolinguistique interactionnelle permet donc de s'intéresser à ce qui se passe dans l'interaction entre des individus socialisés dans des systèmes de communication différents.

Combiner ces trois grands courants (herméneutique, systémique, socioluiguistique) permet donc de penser la rencontre interculturelle à travers les interactions, notamment les pratiques communicatives, ainsi qu'à travers le prisme de la compréhension et l'interprétation, tout en prenant en compte l'apport du passé.

\subsection{Méthodologie}

D'un point de vue méthodologique, cette recherche a pris la forme d'une ethnographie de la rencontre interculturelle. Faire une ethnographie de la rencontre nécessite d'étudier ce qui se passe dans l'interaction - et non seulement les expériences des deux groupes séparément. Il faut alors reconnaitre la nature interactive de la rencontre, et donc l'agentivité des tous ces participant-e-s, ainsi que la nature dynamique des apprentissages et savoirs et donc leur transformation dans le temps.
Il a alors été nécessaire d'enquêter à plusieurs moments de la rencontre et de mettre l'accent sur l'expérimentation avec les stagiaires et les familles d'accueil de leur quotidien.

La recherche a ainsi suivi le parcours de deux groupes de stagiaires participant à des stages QSF au Sénégal, à l'été 2017. La méthodologie combine observations participantes, entretiens individuels et entretiens de groupe, avec les stagiaires et les membres des communautés d'accueil. Les deux groupes participants à cette recherche sont formés de 9 stagiaires dont une ${ }^{4}$ est responsable d'équipe. Les neuf mères d'accueil du premier groupe ont aussi participé à cette recherche. Ce sont elles, dans la famille, qui sont responsables des stagiaires durant la durée du stage. Elles veillent à leur intégration dans le village et reçoivent des directives des partenaires locaux pour s'assurer de leur santé et de leur sécurité. Elles travaillent aussi avec les stagiaires et sont donc présentes à la fois dans leurs maisons et dans leur lieu de travail. Une personneressource d'un des partenaires locaux a aussi participé à la recherche.

En premier lieu, donc, des observations participantes ont été réalisées avant et pendant le stage avec le premier groupe de stagiaires et leurs familles d'accueil. L'observation participante est indissociable de la méthode ethnographique. Selon Maranhao (1986), celle-ci est caractérisée par l'interaction entre l'ethnographe et ses informateurs. Cette relation se vit de façon intersubjective et la réalité qui est coconstruite à travers cette relation est dialogique (Maranhao, 1986). Avec le premier groupe de stagiaires, des observations participantes ont été réalisées avant leur départ (durant les formations prédépart), à leur arrivée à la capitale, à trois moments de leur stage et à leur départ du pays, pour un total de 15 journées d'observation au Sénégal et 3 journées au Québec. Les observations participantes au village étaient aussi réalisées avec les familles d'accueil.

Des entretiens semi-dirigés ont aussi été réalisés avec le premier groupe de stagiaires. L'entretien semi-dirigé est une méthode importante pour accéder aux discours et réflexions des participant-e-s, qui peuvent ensuite être triangulés avec leur pratique. C'est aussi un moment où peuvent être validées ou invalidées certaines observations et interprétations faites par la chercheure. Après avoir 
(in)validé ces interprétations, un sens nouveau peut être construit et négocié entre la chercheure et l'informant-e. Les entretiens effectués étaient d'une durée de 60 à 90 minutes à deux moments différents de leur stage. Les premiers ont eu lieu durant la deuxième semaine de leur arrivée au village et les deuxièmes à 2 semaines de leur départ du village. Chacune des 9 stagiaires a participé à au moins un des entretiens et 5 d'entre elles ont participé aux deux entretiens. Un entretien individuel a aussi été conduit avec une personne-ressource du partenaire local des stagiaires du deuxième groupe.

Des entretiens de groupe ont aussi été réalisés avec le deuxième groupe de stagiaire et avec les mères d'accueil du premier groupe. L'entretien de groupe permet d'être témoin de discussions autour d'un sujet précis, sans attendre que ces discussions aient lieu dans un contexte d'observation participante (Morgan et Spanish, 1984). L'entretien de groupe permet aussi de créer un sentiment de sécurité, puisque les participant-e-s ne sont pas obligés de répondre à toutes les questions (McAllum et Zahra, 2017). Finalement, cette méthode permet d'avoir accès aux interactions entre les participant-e-s, elle permet ainsi de voir comment se construisent les consensus et comment se négocient les désaccords. Ainsi, avec le deuxième groupe de stagiaires, trois entretiens de groupe d'une heure et demie ont été conduits, à trois moments différents de leur stage. La première rencontre $a$ eu lieu après une semaine au village, la deuxième à la moitié du stage et la dernière, le lendemain de leur départ du village. L'entretien de groupe avec les mères d'accueil a eu lieu une fois les stagiaires partis du village et a duré 1 heure.

\section{Résultats}

Adopter ce cadre théorique et méthodologique interculturel m'a permis de mettre en évidence plusieurs dynamiques interculturelles dans la rencontre entre les stagiaires et leurs communautés d'accueil. Nous aborderons ici trois d'entre elles : l'apprentissage sur les systèmes en place, la négociation et la création de nouveaux systèmes hybrides et temporaires, et le développement de savoir-faire interculturels.

\subsection{Apprendre sur les systèmes}

Selon Hottola (2004), le processus d'adaptation interculturel est un mouvement de la confusion vers la compréhension. Selon l'auteur, le modèle de choc culturel, popularisé par Oberg (1969), ne convient pas à la plupart des voyageurs. Entre autres, ce modèle prédit qu'après une première période de «lune de miel », il y aurait une étape « d'hostilité », liée à des émotions négatives. Hottola soutient que ces émotions négatives sont beaucoup moins caractéristiques du processus d'adaptation que l'apprentissage : «When people travel to foreign countries, there often is neither shock nor depression, but they usually get stressed and confused while learning new things or facing unexpected difficulties [...] » (Hottola, 2004, p. 450). Pour contrer la confusion, les individus cherchent alors à être en contrôle et à pouvoir prédire le déroulement des échanges interpersonnels (Hottola, 2014). Ce modèle se conjugue très bien avec une approche herméneutique de la rencontre interculturelle. En effet, dans celui-ci, comme dans la philosophie herméneutique, la rencontre avec l'autre peut causer de la confusion qui peut être résolue par un processus de compréhension. Le modèle de confusion culturelle permet donc de placer le processus de compréhension et d'apprentissage au centre du processus d'adaptation.

Dans le processus d'apprentissage des stagiaires, les savoirs culturels produits - soit les savoirs concernant les particularités culturelles qui ont sens à l'intérieur d'un contexte précis (Bathurst, 2015) - permettent de réduire le sentiment initial de confusion. Produire des savoirs sur les différents systèmes en place (systèmes de communication, de politesse, système familial, etc.) leur permet aussi de devenir compétentes dans ceux-ci. Dès l'or, l'intégration des stagiaires à leur milieu est intimement liée aux savoirs produits.

Ces derniers sont produits à travers différentes interactions. Le processus de production de savoirs «culturels» rappelle le cercle herméneutique de Gadamer. Dans celui-ci, le préjugé laisse place à la compréhension, par l'interprétation, puis revient à une nouvelle précompréhension par la révision. La compréhension n'est alors pas un processus linéaire, mais circulaire (Grondin, 2005). Si le cercle herméneutique se conceptualise normalement comme le rapport entre la personne qui comprend, la compréhension et le compris (Descheneaux, 2014) dans le cas des stagiaires, une grande partie de la compréhension se fait aussi en groupe. 
Avant le départ, les stagiaires et les communautés d'accueil ont des préjugés. Les préjugés, dans la pensée herméneutique, sont conceptualisés comme des idées préalables, comme le point de départ de la compréhension. Ceux-ci vont influencer leur regard sur leur communauté d'accueil. Lorsque les stagiaires arrivent dans leur famille d'accueil «tout est nouveau $»^{5}$, elles se mettent alors à observer et cherchent à construire des connaissances, pour réduire la confusion et l'imprévisibilité. Les premières observations individuelles des stagiaires vont souvent mener à des premiers savoirs perçus comme objectifs. Ces savoirs sont produits en réaffirmant, en nuançant ou en déconstruisant leurs préjugés.

Lorsque le groupe de stagiaires se rencontre, elles ont le réflexe de partager leurs observations, les confrontant à celles des autres. Au sortir de cette mise en commun, les stagiaires vont parfois produire une connaissance de groupe, en révisant les observations individuelles. Celle-ci devient alors le point de départ pour les interprétations futures. Les nouvelles observations qu'elles vont faire vont être produites avec cette attente de compréhension. Les savoirs vont être encore une fois nuancés, infirmés ou confirmés individuellement, puis renégociés en groupe. Il y a ainsi un aller-retour constant entre la précompréhension et une compréhension nouvelle, ainsi qu'entre l'individu et le groupe. Ponctuellement, les savoirs vont aussi être soumis à la validation d'une personne perçue comme médiatrice. Ce peut être les femmes de l'ONG avec laquelle les stagiaires travaillent, des individus dans le village qui parlent français, ou la personne accompagnatrice. Le schéma suivant illustre le processus de production des savoirs culturels.

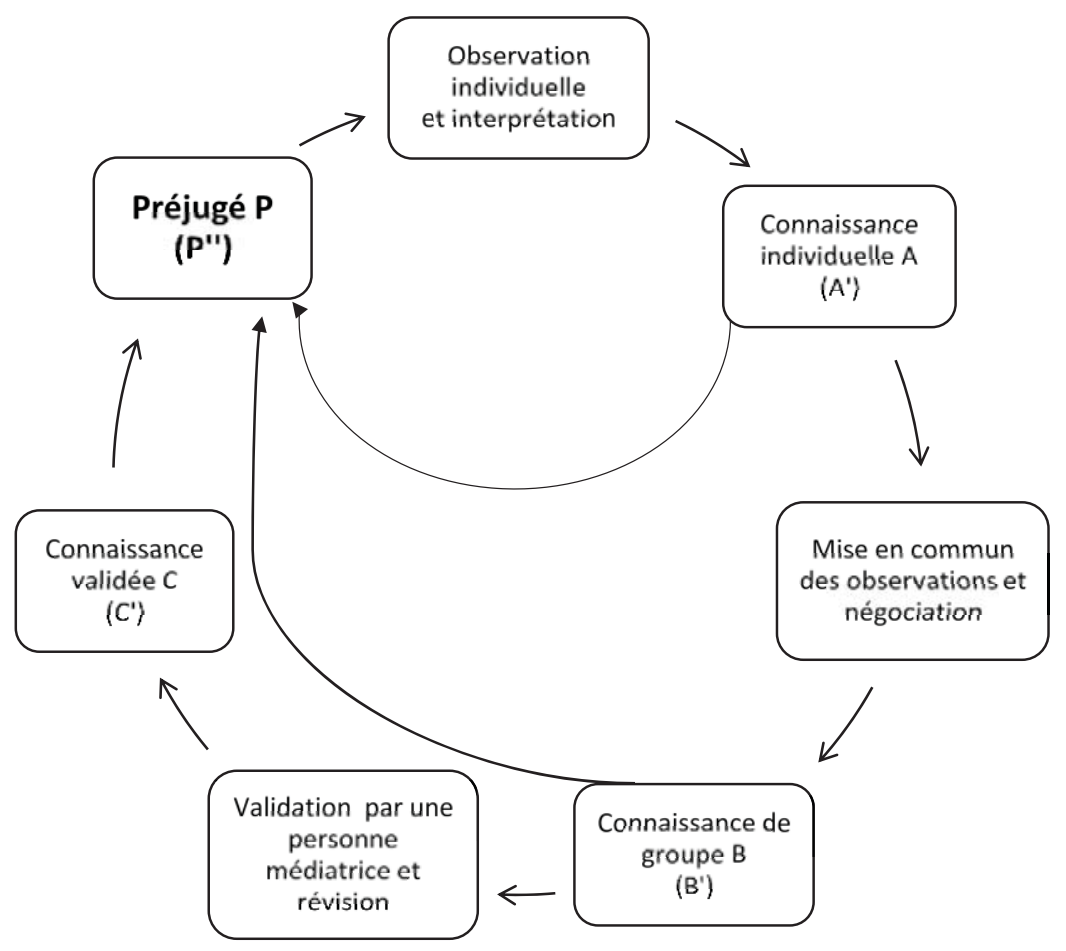

Figure 1 - Cercle herméneutique et production de savoirs culturels Source : Gouin-Bonenfant, 2018, p. 119

Un des cas qui illustre le mieux ce processus est celui des apprentissages autour de la nourriture et de la façon de manger au bol. Dans un nouvel environnement, comprendre les règles autour de la nourriture, des repas et de la commensalité s'avère primordial. C'est l'une des premières préoccupations observées durant les observations participantes. Avant de partir, les stagiaires apprennent, en formation, qu'au Sénégal, on mange à plusieurs autour d'un bol. On leur dit aussi que certaines personnes mangent avec les mains, d'autres avec des cuillères et qu'il ne faut pas utiliser la main gauche. Ces connaissances constituent les préjugés des stagiaires. Ce sont les connaissances préalables qui servent de point de départ à la compréhension des 
pratiques et normes autour de la nourriture. Arrivées dans leurs familles d'accueil, les stagiaires vont faire des observations qui vont confirmer, infirmer ou modifier leurs connaissances préalables. La nature circulaire et collective de la compréhension des stagiaires est apparue rapidement, à travers ce cas. Effectivement, à chaque séjour d'observation conduit au village, il y a eu un moment où les stagiaires ont partagé entre elles des observations et questionnements autour de la nourriture: «Avec qui manges-tu? Les enfants, les femmes, le père, seule? ", "À quelle heure vous mangez? ", « Qui est servi en premier? ", "Qui découpe et distribue les morceaux de viande et de légume? ", ainsi de suite. Les réponses à ces interrogations conduisent à produire des connaissances. Chaque nouvel élément de réponse est une partie qui permet de mieux comprendre le tout. A ma dernière visite des stagiaires au village, les stagiaires sont rendues beaucoup plus compétentes avec les règles, les normes et les pratiques reliées à la nourriture et à la commensalité. Alors qu'au départ, les repas partagés semblaient être un moment un peu stressant, elles savent maintenant ce qui est attendu d'elles et sont ainsi devenues très à l'aise.

Les connaissances culturelles des mères d'accueil sur les stagiaires (sur le Québec, le Canada, l'Occident, ou encore les toubabs $\left.s^{(}\right)$suivent un processus similaire, mais qui s'étale sur plusieurs années. En effet, dans les deux communautés participant à cette étude, cela fait au moins 5 ans que des stagiaires y séjournent. Leur rencontre avec les stagiaires québécoises ne se résume donc pas à une suite d'interactions dans un temps donné ( 2 mois et demi), avec un groupe particulier, mais par une série de rencontres échelonnées sur plusieurs années. Alors que les stagiaires sont en immersion dans un village sénégalais, les mères ne sont pas en immersion dans un contexte québécois. Au fil de l'entretien de groupe avec les mères, quelques éléments ressortent tout de même. Les mères disent avoir appris que les stagiaires aiment travailler et n'aiment pas se faire dire de se reposer. Elles aiment beaucoup les enfants et ne sont pas dérangées par eux. Elles n'aiment pas si les enfants sont punis, surtout par des corrections physiques. Ainsi, les apprentissages que les mères font sur les stagiaires proviennent, comme pour les stagiaires, de besoins. Elles veulent bien accueillir et intégrer les stagiaires et ce sont ces éléments qu'elles vont chercher. Les mères affirment d'ailleurs que d'année en année, les stagiaires s'intègrent de mieux en mieux à la communauté, puisqu'elles-mêmes connaissent de mieux en mieux la culture des stagiaires, facilitant le processus d'intégration.

Le processus de compréhension et d'apprentissage des stagiaires est donc circulaire, collectif et prend son impulsion dans la confusion et la volonté d'intégration. Les savoirs culturels produits à travers celui-ci proviennent d'une série d'interactions. Celles-ci sont de trois types, intragroupes (par la mise en commun, la révision et la négociation), intergroupes (par l'observation ou les questionsréponses) ou avec des personnes médiatrices (par la validation).

\subsection{Négociation et création de nouveaux systèmes hybrides et temporaires}

Alors qu'une grande partie de l'intégration provient de l'apprentissage sur les systèmes en place - puis leur intégration - un autre phénomène a aussi lieu au même moment : la négociation et la création de nouveaux systèmes hybrides et temporaires. Pour illustrer celui-ci, nous prendrons l'exemple des règles de politesse.

À travers leur stage, les stagiaires vont faire des apprentissages sur le système local de politesse. Elles vont par exemple apprendre les comportements polis, comme l'importance des salutations. Elles vont aussi apprendre que certains comportements jugés impolis au Québec, par exemple l'utilisation de l'impératif, n'a pas valeur d'impolitesse au Sénégal. De l'autre côté, les familles d'accueil apprennent que pour les stagiaires québécois, il n'est pas impoli de ne pas s'arrêter longuement pour saluer les personnes que l'on croise dans la rue. Des deux côtés, il y a donc des apprentissages sur ce qui est considéré poli et ce qui ne l'est pas, dans les pratiques communicatives des deux groupes.

Les stagiaires ne vont toutefois pas simplement intégrer le système de politesse qu'elles découvrent. Au même moment se négocient aussi de nouvelles normes d'interaction. Comme nous le rappelle Kecskes (2015), dans les interactions interculturelles, les individus ne font pas partie de la même communauté linguistique (speech community). Malgré les apprentissages d'une part comme de l'autre, les codes et savoirs ne sont pas parfaitement partagés. 
Il doit donc y avoir une forme de co-construction de nouvelles normes et conventions qui permettent de supporter le sens des messages transmis, pour assurer une bonne communication.

Durant l'entretien de groupe avec les mères, nous avons discuté de leur perception des stagiaires et de leur rapport à la politesse. Toutes les mères ont affirmé que les stagiaires étaient parfaitement polies. Après les avoir confrontées avec plusieurs comportements observés chez les stagiaires qui sont contextuellement impolis, les mères ont rigolé et ont répondu qu'évidemment, certains comportements des stagiaires peuvent être considérés comme impolis au Sénégal. Toutefois, elles ont dit savoir que les stagiaires n'agissaient pas par impolitesse. Elles comprennent que les stagiaires ne connaissent pas tous les codes de politesses, alors elles les laissent faire des erreurs qu'elles ne permettraient pas à d'autres membres de la communauté. Les règles de politesse deviennent ainsi moins rigides pour les stagiaires.

Ces dernières essaient par ailleurs de montrer leur respect et leur reconnaissance à leur famille en étant très polies. Parfois, elles surperforment les règles de politesse qu'elles apprennent, installant alors une nouvelle norme plus rigide. Certaines stagiaires racontent qu'elles saluent tous les membres de leur famille, par ordre d'âge, tous les matins, alors qu'elles sont les seules de la famille à procéder ainsi. Elles savent que c'est peut-être un peu exagéré, mais préfèrent être trop polies que pas assez. Une stagiaire me raconte aussi qu'ayant commencé le stage avec cette pratique, elle n'ose maintenant plus la changer. Alors que certaines normes sont moins rigides pour les stagiaires, d'autres pratiques deviennent ainsi plus rigides.

Un autre exemple provient des remerciements. Les stagiaires, s'appuyant sur leurs propres règles de politesse, remercient leurs familles d'accueil en disant constamment « jërëjëf» (« merci », en wolof). Or, dans le système de communication wolof, il n'est pas toujours nécessaire de remercier de cette façon. Ainsi, les mères de famille m'ont expliqué, dans l'entretien de groupe, qu'elles trouvaient très drôle l'habitude des stagiaires de constamment dire « jërëjëf ». Les stagiaires m'ont aussi confié que les mères rigolaient souvent d'elles à ces moments. Toutefois, après quelque temps passé ensemble, les mères se sont aussi mises à dire « jërëjëf » dans les mêmes contextes que les stagiaires.

Ainsi se crée-t-il un nouveau système de politesse. Ce n'est pas celui qui était en place avant l'arrivée des stagiaires et qu'elles auraient simplement intégré, ce n'est pas non plus les pratiques et normes des stagiaires qui auraient été imposées - ni exactement un mélange des deux systèmes. C'est un nouveau système hybride, temporaire, contextuel et dynamique qui se constitue dans une nouvelle communauté de pratique temporaire. D'ailleurs, d'été en été, ces normes sont transformées, puisque de nouvelles stagiaires arrivent.

Finalement, ce processus de négociation rappelle la fusion des horizons de Gadamer. L'horizon est modelé par les traditions des individus et est « [...] le champ de vision qui comprend et inclut tout ce que l'on peut voir d'un point précis » (Gadamer, 1996, p. 324). Dans la fusion, les traditions « se métamorphosent pour faire place à autre chose » (Grondin, 2005, p. 5). C'est effectivement le processus qui est à l'œuvre dans la rencontre entre les stagiaires et leurs familles d'accueil : dans le processus d'apprentissage des uns sur les autres, l'écart entre les traditions est réduit, alors qu'il se crée de nouveaux systèmes hybrides et temporaires qui permettent une meilleure communication et une meilleure compréhension entre les deux groupes. Il ne s'agit pas d'affirmer qu'il y a fusion des horizons, mais plutôt qu'un processus de réduction des écarts de compréhension est entamé, à l'aide de la création d'un langage commun.

\subsection{Les savoir-faire interculturels comme habitude}

Nous venons d'identifier deux dynamiques qui ont lieu simultanément. Premièrement, l'apprentissage conscient d'éléments de la «culture» locale et des différents systèmes que les stagiaires tentent d'intégrer. Parallèlement, il y a aussi co-construction de nouvelles normes et pratiques qui se constituent comme de nouveaux systèmes hybrides et temporaires. Dans un troisième temps, nous pouvons aussi ajouter le développement de savoir-faire interculturels, c'est-à-dire les savoirs qui ne portent pas sur un contexte culturel précis, mais sur les contextes interculturels. Si les savoirs culturels permettent d'être 
compétent-e dans un contexte culturel donné, les savoirs interculturels permettraient d'être compétent-e dans les contextes interculturels.

Un des savoir-faire identifiés est la navigation entre les échelles individuelles et culturelles. Au départ, les stagiaires attribuent une très grande partie des comportements des membres de leur famille d'accueil à la culture. Après avoir remarqué que son père d'accueil était peu bavard, une stagiaire me dira qu'elle a compris que les hommes au Sénégal parlaient peu. Ainsi, la lentille d'analyse sur la réalité autour d'elles est celle de «la culture». Au fur et à mesure que le stage avance, cette lentille tend à changer. C'est qu'en discutant avec le groupe, les stagiaires se rendent compte que certains des savoirs qu'elles construisent ne tiennent pas. En fait, si son père d'accueil est peu bavard, dira la stagiaire dans un deuxième entretien, c'est probablement en raison de sa personnalité et des dynamiques familiales.

À la suite de cette discussion, cette question est amenée dans un entretien de groupe réalisé avec l'autre groupe de stagiaires. Toutes les stagiaires sont alors d'accord pour dire qu'elles avaient eu ce réflexe au début de leur séjour, mais que celui-ci a été déconstruit au fur et à mesure du stage. Elles expliquent ce changement de logique par la durée et par l'immersion. Les stagiaires réalisent que les individus sont influencés par les mêmes normes culturelles, mais que leurs comportements vis-à-vis celles-ci sont différents. Les stagiaires m'expliquent aussi qu'elles ont réalisé que de famille en famille, les dynamiques sont différentes et que ce qu'une stagiaire observe dans sa famille «ce n'est pas coulé dans le béton». Finalement, une stagiaire dit qu'il faut aussi appliquer cette logique à l'échelle du village : ce qu'elles pensent être «culturel», m'affirme-t-elle, c'est la «culture du village» et ça ne peut pas être généralisé à tout le Sénégal.

Ce qui ressort de cette discussion, c'est que les stagiaires, par leur séjour prolongé dans le même village, ont appris à naviguer entre les échelles individuelles et groupales. Ce savoir-faire interculturel leur permet aussi d'abandonner (au moins de réduire) la lecture « culturaliste » d'abord adoptée, où ce qui est observé est tout de suite attribué à la culture. Elles se rendent par ailleurs compte que jamais elles ne feraient la même chose au Québec. Une des stagiaires conclut :
"Jamais à la maison, mettons au Québec, il y a quelqu'un qui va dire quelque chose et moi je vais être comme : "ça, c'est parce qu'il est québécois" ».

À travers le partage, la négociation et la révision de leurs savoirs produits individuellement, certaines erreurs d'interprétation récurrentes se révèlent, telles que l'interprétation culturaliste. Sans prendre nécessairement pleinement conscience des logiques derrière ces erreurs, il se crée toutefois, dans la révision, de nouvelles habitudes d'apprentissage qui, à force de répétitions, se matérialisent comme des savoir-faire.

Plusieurs études posent l'hypothèse - ou arrivent à la conclusion - que la compétence interculturelle est acquise par l'expérience internationale et l'interaction interculturelle (Bartel-Radic, 2014). Cela semble logique, puisque la compétence est « une intelligence de l'action » et que l'expérience pratique est un élémentclé de l'apprentissage (Gratton, 2009, p. 93). Bennett (2013) met toutefois en garde contre la réification de la compétence interculturelle. Il serait difficile de la mesurer comme une chose « que l'on a » puisque c'est plutôt « quelque chose que l'on fait ». L'idée n'est pas ici de conclure si l'expérience internationale et interculturelle mène nécessairement au développement de la compétence interculturelle comme une chose acquise, mais de voir comment l'expérience particulière vécue par les stagiaires et leurs familles d'accueil permet le développement de savoir-faire interculturels qui leur permettent de mieux naviguer à travers les complexités de la rencontre interculturelle.

\section{Conclusion}

Cet article a mis de l'avant la pertinence d'une approche théorique et méthodologique interculturelle pour l'étude de la relation entre les bénévoles et les communautés hôtes dans le bénévolat international. La littérature sur ce thème se retrouve le plus souvent dans une opposition entre des postures enthousiastes et critiques. Une posture interculturelle transforme l'objet d'étude du résultat de la rencontre à son processus. Elle reconnait l'agentivité de tous les acteurs et actrices de la rencontre et reconnait l'importance des contextes sur les interactions. Finalement, elle laisse place à l'étude d'une multitude de dynamiques interculturelles souvent laissées de côté dans les études sur le bénévolat international. 
Pour illustrer l'apport de cette posture, une étude de cas sur la relation entre les stagiaires et leur famille d'accueil dans les stages Québec Sans Frontières au Sénégal a été présentée. L'étude s'intéressait particulièrement aux savoirs culturels et interculturels (co-)produits dans la rencontre, ainsi que leur relation aux processus d'adaptation et d'intégration des stagiaires. L'approche proposée a permis de mettre en lumière trois dynamiques. Premièrement, nous avons abordé la production de savoirs culturels comme une façon de réduire la confusion initiale liée à la mobilité. Ces savoirs sont produits à travers plusieurs interactions et leurs trajectoires circulaires rappellent le cercle herméneutique. Alors que ces savoirs sur l'autre sont produits (des deux côtés), il y a simultanément une hybridation qui se fait. Nous avons illustré celle-ci avec les règles de politesse. À travers la rencontre entre les deux groupes, un nouveau système hybride se crée. Les stagiaires ne font pas qu'intégrer un système de règles et de normes de politesse qui serait figé. Au contraire, au fil des interactions, certaines normes se rigidifient, d'autres s'assouplissent et de nouvelles émergent. Finalement, nous avons aussi abordé le développement de savoirfaire interculturels. Ceux-ci sont le fruit de nouvelles habitudes d'apprentissage qui se créent à travers le processus de négociation et de révision des savoirs culturels produits. Nous avons présenté le cas de la navigation entre les échelles individuelles et groupales.

Malgré que la posture interculturelle permette une reconnaissance de l'agentivité de tous les acteurs et actrices, l'étude de cas présentée s'est surtout intéressée aux points de vue des bénévoles. Comme nous l'avons mentionné, les savoirs culturels et interculturels produits par les familles d'accueil se font sur la longue durée, au fil des années, avec les différents groupes de stagiaires qu'elles reçoivent. La durée du terrain - réalisé dans le cadre d'une maitrise - ne permettait pas cette analyse longitudinale. Il serait toutefois très pertinent de réaliser une telle étude avec les familles d'accueil.

\section{NOTES}

1 Des articles critiques sont parus dans plusieurs journaux, comme The New York Times, Libération, Le Monde, Huffington Post et The Guardian (Biddle, 2017; Biddle, Ernst, Johnson, Richter et Zakaria, 2014; Dupont, 2018; Jenkin, 2015; Rousseau, 2016).

2 Cet article reprend certaines parties du mémoire «Du choc à la confusion : la rencontre interculturelle dans les stages Québec Sans Frontières au Sénégal» (2018) de l'auteure.

3 Ce cadre théorique est inspiré principalement du modèle théorique interculturel développé par le Laboratoire de recherche en relations interculturelles (LABRRI).

4 Comme la majorité des stagiaires sont des femmes, le féminin sera utilisé dans cet article pour référer aux stagiaires.

5 Expression entendue à plusieurs reprises par les stagiaires.

6 Terme qui signifie à la fois étrangers, blancs et/ou européens.

\section{RÉFÉRENCES}

Agar, M. H. (1982). Toward an Ethnographic Language. American Anthropologist, 84(4), 779-795. doi: 10.1525/aa.1982.84.4.02a00030

Bartel-Radic, A. (2014). La compétence interculturelle est-elle acquise grâce à l'expérience de mobilité? Management international, 18, 194-211. doi: 10.7202/1027873ar

Bathurst, L. (2015). Culture Specific/Culture General. Dans J. M. Bennett (Éd.), The SAGE Encyclopedia of intercultural Competence. (pp. 211-213). Thousand Oaks, CA: SAGE Publications, Inc.

Bathurst, L. et La Brack, B. (2010). Shifting the locus of intercultural learning. Intervening Prior to and After Student Experiences Abroad. Dans M. Vande Berg, M. Paige et K.H. Lou (Éds), Student Learning Abroad: What Your Students Are Learning, What They're Not, and What You Can Do About It. (pp. 261-283). Sterling, VA: Stylus Publishing.

Biddle, P. (6 décembre 2017). The problem with little white girls, boys and voluntourism. Huffington Post. Repéré à https://www.huffpost.com/entry/little-white-girls-voluntourism_b_4834574 
Biddle, P., Ernst, A., Johnson, C., Richter, L. et Zakaria, R. (29 avril 2014). Can voluntourism make a difference? The New York Times. Repéré à https://www.nytimes.com/roomfordebate/2014/04/29/can-voluntourism-make-a-difference

Broad, S. (2003). Living the Thai life_-a case study of volunteer tourism at the Gibbon rehabilitation project, Thailand. Tourism Recreation Research, 28(3), 63-72. doi: 10.1080/02508281.2003.11081418

Butcher, J. (2003). The moralisation of tourism: sun, sand-- and saving the world? New York, NY: Routledge.

Butcher, J. et Smith, P. (2010). «Making a difference »: volunteer tourism and development. Tourism recreation research, 35(1), 27-36. doi: 10.1080/02508281.2010.11081616

Colleyn, J. et Devillez, F. (2009). Le tourisme et les images exotiques. Cabiers d'Études Africaines 49(193-194), 583-594. Repéré à http://journals.openedition.org/etudesafricaines/18839

Coghlan, A. et Gooch, M. (2011). Applying a transformative learning framework to volunteer tourism. Journal of Sustainable Tourism, 19(6), 713-728. doi: 10.1080/09669582.2010.542246

Crabtree, R. D. (1998). Mutual empowerment in cross-cultural participatory development and service learning: Lessons in communication and social justice from projects. Journal of Applied Communication Research, 26(2), 182-209. doi: 10.1080/00909889809365501

Daldeniz, B. et Hampton, M. (2010). Charity-based Voluntourism Versus « Lifestyle » Voluntourism: Evidence from Nicaragua and Malaysia. [Document de travail]. Canterbury, Royaume-Uni: University of Kent.

Debono, M. (2012). Herméneutique interculturelle et DLC: quelques fondements théoriques pour une didactique des représentations. Dans M. Debono et C. Goï (Éds), Regards interdisciplinaires sur l'épistémologie du divers : Interculturel, herméneutique et interventions didactiques. (pp. 131-168). Fernelmont, Belgique: Éditions Modulaires Européennes.

Descheneaux, H. (2014). L’herméneutique phénoménologique de Gadamer. Cabiers du Cirp, 5, 1-99.

Dettweiler, U., Ünlü, A., Lauterbach, G., Legl, A., Simon, P. et Kugelmann, C. (2015). Alien at home: Adjustment strategies of students returning from a six-months over-sega's educational programme. International Journal of Intercultural Relations, 44, 72-87. doi: 10.1016/j.ijintrel.2014.10.005

Dupont, M. (25 juin 2018). «Volontourisme» : le juteux business de l’humanitaire sur catalogue. Le Monde. Repéré à https://www.lemonde.fr/idees/article/2018/06/24/volontourisme-le-juteux-business-de-l-humanitaire-surcatalogue_5320317_3232.html

Farah, I. (1997). Ethnography of Communication. Dans H. Hornberger et D. Corson (Éds), Encyclopedia of Language and Education. (pp. 125-133). Dordrecht: Springer Netherlands.

Gadamer, H. (1996). Vérité et méthode. Les grandes lignes d'une herméneutique philosophique. [1960]. Paris, France: Éditions du Seuil.

Gagnon, M.-J. (21 janvier 2016). Que penser du volontourisme? Avenues.ca. Repéré à https://avenues.ca/chroniques/quepenser-du-volontourisme/

Gius, C. (2012). Encountering the other, or encountering the self? The construction of otherness into volunturism experiences. Dans O. McGarry et A. Stasiewicz-Bienkowska (Éds.), Landscapes of (Un) Belonging: Reflections of Strangeness and Self. (pp. 1-12). Oxford: ID Press.

Gouin-Bonenfant, M. (2018). Du choc à la confusion: La rencontre interculturelle dans les stages Québec Sans Frontières (Mémoire de maitrise inédit). Université de Montréal, QC.

Gratton, D. (2009). L'interculturel pour tous: une initiation à la communication pour le troisième millénaire. Anjour, QC: Éditions Saint-Martin.

Grondin, J. (2002). Gadamer's basic understanding of understanding. Dans R. Dostal (Éd.), The Cambridge companion to Gadamer. (pp. 36-51). Cambridge: Cambridge University Press.

Grondin, J. (2005). La fusion des horizons. La version gadamérienne de l'adaequatio rei et intellectus. Archives de philosophie, 68(3), 401-418. doi: 10.3917/aphi.683.0401

Grondin, J. (2017). L’herméneutique (4e éd.). Paris, France: Presses universitaires de France.

Gumperz, J. (2005). Interactional sociolinguistics: A personal perspective. Dans D. Tannen, H. Hamilton et D. Schiffrin (Éds), The handbook of discourse analysis. (2e éd., pp. 215-228). Oxford: Blackwell Publishing 
Guttentag, D. (2009). The possible negative impacts of volunteer tourism. International journal of tourism Research, 11, 537-551. doi: $10.1002 /$ jtr

Hachey, I. (9 janvier 2016). Le business du volontourisme : L’humanitaire imaginaire. La Presse. Repéré à http://plus. lapresse.ca/screens/98b8c227-78a9-4bb8-8071-77c6d0570f59_7C_I2rIIq53D6k0.html

Heller, M. (2013). Gumperz and Social Justice. Journal of Linguistic Anthropology, 23(3), 192-198. doi: 10.1111/jola.12026

Higgins-Desbiolles, F. (2003). Reconciliation Tourism: Tourism Healing Divided Societies! Tourism Recreation Research, 28(3), 35-44. doi: 10.1080/02508281.2003.11081415

Hottola, P. (2004). Culture confusion: Intercultural Adaptation in Tourism. Annals of Tourism Research, 31(2), 447-466. doi: 10.1016/j.annals.2004.01.003

Hymes, D. (1964). Introduction: Toward ethnographies of communication. American Anthropologist, 66(6), 1-34. doi: 10.1525/aa.1964.66.suppl_3.02a00010

Jafari, J. (2001). The scientification of tourism. Dans V. L. Smith et M. Brent (Éds.), Hosts and guests revisited: Issues of the 21st century (pp. 28-41). Elmsford, NY: Cognizant.

Jenkin, M. (21 mai 2015). Does voluntourism do more harm than good? The Guardian. Repéré à https:// www.theguardian.com/voluntary-sector-network/2015/may/21/western-volunteers-more-harm-than-good

Kecskes, I. (2015). Intercultural impoliteness. Journal of Pragmatics, 86, 43-47. doi: 10.1016/j.pragma.2015.05.023

Lawrence, G. (25 avril 2016). Barbie Savior, ou les dérives du volontourisme. L'actualité. Repéré à https://lactualite.com/ art-de-vivre/2016/04/25/barbie-savior-ou-les-derives-du-volontourisme/

Lyons, K. et Wearing, S. (2008). Journeys of discovery in volunteer tourism: international case study perspectives. Wallingford: CABI Publishing.

Lyons, K., Hanley, J., Wearing, S. et Neil, J. (2012). Gap year volunteer tourism. Myths of Global citizenship? Annals of tourism research, 39(1), 361-378. doi: 10.1016/j.annals.2011.04.016

Maranhao, T. (1986). The hermeneutics of participant observation. Dialectical Anthropology, 10(3-4), 179-188. doi : 10.1007/BF02343112

Marc, E. et Picard, D. (2000). L'école de Palo Alto : Un nouveau regard sur les relations bumaines. Paris, France: Éditions Retz.

McAllum, K. et Zahra, A. (2013). Constructing «Them » and « Us » : Host Communities' Perspectives of Voluntourist Identities. Dans M. Kramer, L. Gossett et L. K. Lewis (Éds), Volunteering and intercultural communication: studies from multiple contexts. (Vol. 2, pp. 109-128). New York: Peter Lang.

McAllum, K. et Zahra, A. (2017). The positive impact of othering in voluntourism: The role of the relational other in becoming another self. Journal of International an Intercultural Communication 47(5), 392-398. doi: 10.1080/17513057.2017.1280179.

Mcgehee, N. G. (2002). Alternative tourism and social movements. Annals of Tourism Research, 29(1), 124-143. doi: 10.1016/S0160-7383(01)00027-5

Mcgehee, N. G. et Santos, C. A. (2004). Social change, discourse and volunteer tourism. Annals of Tourism Research, 32(3), 760-779. doi: 10.1016/j.annals.2004.12.002

McIntosh, A. J. et Zahra, A. (2007). A Cultural Encounter through Volunteer Tourism: Towards the Ideals of Sustainable Tourism? Journal of Sustainable Tourism, 15(5), 541-556. doi: 10.2167/jost701.0

Morgan, D. et Spanish, M. (1984). Focus groups: A new tool for qualitative research. Qualitative Sociology, 7(3), 253-270. doi: $10.1007 /$ BF00987314

Mostafanezhad, M. (2014). Volunteer tourism and the popular humanitarian gaze. Geoforum, 54, 111-118. doi: 10.1016/ j.geoforum.2014.04.004

Murphy-Lejeune, E. (2000). Mobilité internationale et adaptation interculturelle : les étudiants voyageurs européens. Recherche \& Formation, 33, 11-26. doi: 10.3406/refor.2000.1614 
Pastran, S. H. (2014). Volunteer Tourism : A Postcolonial Approach. University of Saskatchewan Undergraduate Research Journal, 1(1), 45-57. doi: 10.32396/usurj.v1i1.30

Québec Sans Frontières (QSF). (2018). Nos valeurs. Repéré à http://www.mrif.gouv.qc.ca/qsf/index.asp?Page= programme\&SousPage $=$ valeurs

Raymond, E. M. et Hall, C. M. (2008). The Development of Cross-Cultural (Mis) Understanding Through Volunteer. Journal of Sustainable Tourism, 16(5), 530-543. doi: 10.2167/jost796.0

Rousseau, N. (15 août 2016). Tourisme humanitaire: la vraie fausse pitié. Libération. Repéré à https://www.liberation.fr/ planete/2016/08/15/tourisme-humanitaire-la-vraie-fausse-pitie_1472579

Simpson, K. (2004). « Doing development »: The gap year, volunteer-tourists and a popular practice of development. Journal of International Development, 16(5), 681-692. doi: 10.1002/jid.1120

Sin, H. L. (2009). Volunteer Tourism-"Involve Me and I Will Learn"? Annals of Tourism Research, 36(3), 480-501. doi 10.1016/j.annals.2009.03.001

Sin, H. L. (2010). Who are we responsible to? Locals' tales of volunteer tourism. Geoforum, 41(6), 983-992. doi: 10.1016/j.geoforum.2010.08.007

Stronza, A. (2001). Anthropology of Tourism: Forging New Ground for Ecotourism and Other Alternatives. Annual Review of Anthropology, 30, 261-283. doi: 10.1146/annurev.anthro.30.1.261

Tannen, D. (2005). Interactional Sociolinguistics as a resource for Intercultural Pragmatics. Intercultural Pragmatics, 2(2), 205209. doi: 10.1515/iprg.2005.2.2.205

Theerapappisit, P. (2009). Pro-poor Ethnic Tourism in the Mekong: A Study of Three Approaches in Northern Thailand. Asia Pacific Journal of Tourism Research, 14(2), 201-221. doi: 10.1080/10941660902847245

Van den Berghe, P. (1980). Tourism as ethnic relations: A case study of Cuzco, Peru. Ethnic and Racial Studies, 3(4), 375-39. doi: 10.1080/01419870.1980.9993312

Venne, A. (22 février 2016). Volontourisme ou coopération internationale? Huffington Post. Repéré à https://quebec. huffingtonpost.ca/un-seul-monde/volontourisme-aide-humanitaire-industrie-ethique-voyage-tourisme_b_9274696.html

Ward, C. et Kennedy, A. (1999). The measurement of sociocultural adaptation. International Journal of Intercultural Relations, 23(4), 659-677. doi: 10.1016/S0147-1767(99)00014-0

Wearing, S. (2001). Volunteer tourism : experiences that make a difference. Wallingford: CABI Publishing.

Wearing, S. et Mcgehee, N. G. (2013). Volunteer tourism: A review. Tourism Management, 38, 120-130. doi: 10.1016/ j.tourman.2013.03.002 